\title{
Al badge ci siamo ormai abituati, a beggiare e alla beggiatura no
}

\author{
Riccardo Gualdo
}

PUBBLICATO: 25 AGOSTO 2020

\section{Quesito:}

Molti lettori chiedono notizie sulla parola badge e sulla legittimità di usare nella nostra lingua gli adattamenti basati su badge, come i verbi badgeare, badgi(e)are o beggiare, e il sostantivo beggiatura.

\section{Al badge ci siamo ormai abituati, a beggiare e alla beggiatura no}

$\mathrm{L}^{\prime}$

anglicismo badge, entrato in circolazione già da quasi quarant'anni, era stato già esaminato nel volume Inglese-italiano 1 a 1. Tradurre o non tradurre le parole inglesi?; in quell'occasione che sembra ormai molto lontana nel tempo, vista la rapidità con cui gli anglicismi attecchiscono nel nostro vocabolario - Claudio Giovanardi, Alessandra Coco e chi scrive avevano concluso, con cauto ottimismo, che badge avrebbe potuto "essere rimpiazzato senza sforzi eccessivi" dai suoi equivalenti italiani (cfr. Giovanardi-Gualdo-Coco 2008, pp. I47-I48).

Oggi forse saremmo meno convinti; ma andiamo con ordine.

Badge è un prestito non adattato nella grafia, identica a quella inglese, ed è ritenuto da tutti i vocabolari italiani che lo registrano come morfologicamente invariabile: occasionale, ma raro nell'italiano di tutti i giorni, è il plurale badges. La sequenza grafica $d g$ non è normale nell'ortografia italiana per rendere il suono /dz/, un'affricata prepalatale sonora simile (non proprio identica) alla $g$ di gente. È tuttavia una grafia non insolita, se si pensa a un altro anglicismo, bridge, notissimo sia nel suo significato più concreto ('ponte', come il London bridge), sia come gioco di carte (la parola circola nella nostra lingua almeno dall'inizio del secolo scorso). Un po' più complicata la pronuncia della a: il fonema /x/ (in inglese britannico e americano è lo stesso: /bæd3/), non coincide né con una e - aperta o chiusa - dell'italiano, né con una $a$. Di fatto, la pronuncia italiana più diffusa è /bedz/, ma non è infrequente sentire /beid3/, forse per influsso di parole come age 'età', per es. in prodotti antiage, e stage 'periodo di tirocinio' (ricordo però che con questo significato il termine stage è francese e quindi andrebbe pronunciato /'stazə/); ma è un uso erroneo, cosi come lo sarebbe un pur possibile /badz/, trainato dalla grafia. Il genere di badge è maschile, ma in rete si trovano anche, molto più rare, attestazioni di "la badge", "le badge", probabilmente indotte dall'associazione con tessera.

Veniamo al significato, o meglio ai significati, perché i dizionari italiani ne registrano almeno tre: I. 'cartellino di riconoscimento che si appende o si fissa a una giacca o a un abito', per esempio in occasione di convegni, fiere, e simili; in genere è plastificato o protetto da plastica trasparente; 2. 'distintivo, metallico o di plastica, per vari usi'; sono stati chiamati badge le spille di latta, quasi sempre rotonde, usate per distinguere i partecipanti a una manifestazione politica, o anche quelle con disegni o scritte umoristiche o contestatrici; 3. 'tesserino magnetizzato di identificazione, per vari usi', soprattutto quello che impiegati e funzionari usano per registrare l'entrata o l'uscita dal posto di lavoro, avvicinandolo o passandolo all'interno di un'apposita apparecchiatura. Assai più marginale, ma interessante, una quarta accezione dell'anglicismo, specialistica e non registrata dai repertori che ho potuto consultare: in situazioni di lavoro che espongono a radiazioni, un (photographic) film badge dosimeter è un piccolo strumento o che contiene un ritaglio quadrato di pellicola sensibile alle 
radiazioni; il lavoratore lo indossa al polso o alla cintura e dal variare del colore della pellicola può capire se ha subito radiazioni o no, e misurarne eventualmente la quantità (dosimeter). Nei testi specialistici italiani si trova nella forma abbreviata film badge o, ancor più semplicemente, badge: in questo caso, il badge è lo strumento, che ha l'aspetto e le dimensioni di una tesserina.

Stando ai nostri dizionari, i primi due significati sembrerebbero i più antichi: i dizionari dell'uso ed etimologici (GRADIT, l'Etimologico) li datano al I98 nei primi anni 20oo. In realtà, ricorrendo a Google libri, è possibile trovare attestazioni del significato 3. già nei primi anni Settanta del secolo scorso, per esempio nel primo volume della "Rivista dell'informazione" (va osservato che il periodico è bilingue, italiano e inglese, e che nel testo italiano badge è scritto tra virgolette); riporto un contesto:

Presso tutte le Unità che adotteranno il sistema proposto, verranno aboliti i cartellini orologio. Per la rilevazione delle presenze ogni operaio avrà a disposizione un "badge".

Come ho avuto modo di scrivere tempo fa (cfr. Gualdo 20I0, pp. I53-I86), poche isolate attestazioni di un anglicismo che ha una storia antica nella lingua d'origine non consentono di valutarne la reale circolazione tra i parlanti. Sono molte le parole "a scoppio ritardato" che per anni, se non per decenni, non escono da un ristretto ambito d'uso (tecnici, scienziati, specialisti di qualche settore professionale), e poi, per motivi diversi (campagne promozionali, adozione in testi istituzionali), "esplodono" raggiungendo una massa considerevole di persone, e giustificando la registrazione nei dizionari. E dunque è proprio il terzo significato di badge quello meglio radicato nell'uso; sia perché l'oggetto a cui si riferisce è diventato senz'altro di più "alta disponibilità" nella vita di tutti i giorni per ricorrere a una categoria introdotta da Tullio De Mauro a proposito di una parte del "vocabolario di base" -, sia perché, probabilmente, ha una maggiore funzionalità semantica, dato che serve a distinguere il tesserino di riconoscimento dalla carta (o la card) di negozi e supermercati e dalla tessera dei mezzi pubblici.

Dunque badge è senz'altro entrato pienamente nell'uso comune, almeno nel significato 3.; se sia giusto o no usarlo, dipende dalla sensibilità di ognuno di noi. Ad aiutarci nella scelta possono essere alcune considerazioni ulteriori.

Per i parlanti inglesi e americani badge ha soprattutto i significati 2. e i., cioè quelli di 'distintivo, stemma' e di 'cartellino di riconoscimento'. Lorigine di badge è incerta: il più autorevole vocabolario dell'inglese britannico, l'Oxford English Dictionary, propone come prima accezione della parola 'Un distintivo, emblema o marchio usato originariamente per identificare un cavaliere o distinguere i suoi seguaci, oggi indossato come segno di un ufficio o di un impiego riconosciuto, come simbolo dell'appartenenza a qualche associazione, ecc.' (traduzione mia); come primo esempio riporta alcuni versi tratti da una versione medio-inglese del Romanzo di Alessandro, datata al I350; vi sono menzionati "bages and baners" dei cavalieri, cioè 'stemmi e bandiere', e bage è appunto l'antica grafia del moderno badge. In inglese esistono anche il verbo transitivo (to) badge ('marcare, distinguere con un badge'), il sostantivo badger ('chi porta o indossa un badge') e diversi composti a due termini, come badge-ticket 'ingresso con un badge', per es. alle corse dei cavalli.

Nell'accezione più comune, il badge è un distintivo: metallico, come la stella dello sceriffo, ma anche di stoffa, cucito sul risvolto della giacca, su altri indumenti, come una sciarpa, o su borse e tascapane; può poi essere anche la spilla di riconoscimento dell'appartenenza a un'associazione, a un club Questo secondo tipo di distintivo è anche detto button badge, cioè 'distintivo a bottone', o round badge 'distintivo rotondo', ma anche, più semplicemente, pin 'spilla'. 
Possibili, come per marchio o emblema in italiano, gli usi traslati: nell'Enrico IV di Shakespeare il fegato bianco e livido è il marchio (badge) della pusillanimità e della codardia. L'Oxford Dictionary registra anche l'uso di badge in marineria, per lo stemma fissato sulla poppa di piccole imbarcazioni.

Il badge può infine anche essere un tesserino o un cartello di riconoscimento: il blue badge, rilasciato dalle autorità dei trasporti, è il documento che consente ai veicoli che trasportano persone con disabilità di parcheggiare in spazi riservati; la stessa parola indica tanto la tessera plastificata quanto il permesso orario che si mette in mostra su un vetro o sul cruscotto.

Per tutte queste accezioni esistono validi equivalenti italiani: stemma, distintivo, spilla, tessera o tesserina/tesserino, cartellino; per il simbolo di una squadra sportiva si può usare ovviamente anche scudetto, che rivela l'antica origine cavalleresca. È chiaro che la polisemia nella lingua d'origine e in quella d'arrivo, la relativa antichità d'uso, la brevità della parola e la grafia e la pronuncia non troppo ostiche giocano a favore di un'assimilazione della parola in italiano; ma conta soprattutto, come al solito, il valore connotativo degli anglicismi, che sono avvertiti come alternative più eleganti e prestigiose dei loro omologhi italiani, anche quando questi ultimi funzionano benissimo. Contrastare l'uso della parola, come fa una professoressa che ci ha scritto, è legittimo; soprattutto, direi, per incoraggiare all'uso dei sinonimi italiani, piuttosto che per sbarrare la strada al nostro badge.

Fin qui per badge. Che dire dei suoi adattamenti?

Già nel 2008 avevamo annotato l'uso di beggiare, più nel parlato che nello scritto, per 'vidimare' o meno burocraticamente - 'passare, strisciare' un cartellino magnetico di riconoscimento. Nel libro di Antonio Giangrande Italia allo specchio: il DNA degli italiani. Anno 2020, pubblicato nel 2019 dall'associazione "Contro tutte le mafie", trovo il resoconto di una nota vicenda di qualche anno fa:

Sanremo: il vigile in mutande dopo l'assoluzione: "Rivoglio il mio lavoro". [...] L'ex vigile diventato virale per aver
beggiato in mutande rivuole il suo posto in Comune, dopo essere stato assolto [...] nel procedimento
contro i presunti "furbetti del cartellino". L'uomo era stato pizzicato a beggiare in mutande e le sue
immagini avevano fatto il giro del Paese. [...] Nonostante tre anni fa sia stato approvato il decreto anti-
fannulloni, molti furbetti del cartellino continuano purtroppo a "beggiare" irregolarmente.

Se non capisco male, Giangrande trascrive il testo di una puntata del programma televisivo "Le Iene"; dunque siamo di fronte alla versione scritta di un discorso orale. L'uso scritto di beggiare è piuttosto raro: un sondaggio in Google libri, limitato alle pubblicazioni in lingua italiana e alla sola forma dell'infinito presente, restituisce una decina di risultati per beggiare, nessuno per bedgiare e bedgeare, tre o quattro per badgiare. L'altra cosa degna di nota è l'associazione di beggiare all'espressione giornalistica "furbetti del cartellino", riformulazione scherzosa del pure scherzoso "furbetti del quartierino", la cui storia è stata ricostruita da Valeria Della Valle qualche anno fa (cfr. Della Valle 2006). Beggiare, insomma, corrisponde a 'timbrare il cartellino', ed è proprio l'associazione a cartellino che lo rende davvero riconoscibile, trasparente per il parlante.

La grafia beggiare risponde correttamente alla pronuncia, e secondo me è preferibile alle varianti badgeare, bedgeare e simili, diversamente da come ritiene un lettore. L'antica parola medievale inglese bage - quale che sia la sua origine - ricorda da vicino gage, adattamento francese del germanismo wadi, che ha prodotto già nel Duecento gli italiani gaggio, guaggio 'pegno, ostaggio' e, tramite engagier, il verbo ingaggiare 'dare in pegno' e poi 'iniziare una battaglia' (cfr. Cella 2003, pp. 4I4-4I5 e 443): l'adattamento beggiare mostra la forza di assimilazione del nostro sistema fono-morfologico, e non disprezzerei - pur preferendo le alternative autoctone che ho già ricordato - nemmeno un eventuale *beggio (direi cone aperta, come le forme rizotoniche di beggiare). Sul piano semantico, invece, la 
persistenza di cartellino suggerisce con chiarezza i migliori equivalenti italiani: timbrare il cartellino mi sembra più chiaro di vidimare il badge, anche se il cartellino è magnetico e non è, a rigore, timbrato.

Direi altrettanto per beggiatura: meglio in questa forma che con $d g$. La parola non ha un aspetto seducente, ma non cozza con il nostro vocabolario (ombreggiare e ombreggiatura sono "brutte"?); osservo semmai che non è comunissima nell'uso scritto: una rapida ricerca in rete restituisce solo 234 risposte per beggiatura e poco più di Iooo per il plurale beggiature. Non mi pare, quindi, che si possa dire che il verbo e i suoi derivati sono ormai entrati nell'uso comune, come ritiene qualcuno: ancora una volta il martellamento mediatico e la moda angloamericaneggiante producono una distorsione percettiva che contrasta con la realtà dell'uso.

\section{Nota bibliografica:}

- Cella 2003: Roberta Cella, I gallicismi nei testi dell'italiano antico, Firenze, Accademia della Crusca, 2003 .

- Della Valle 2006: Valeria Della Valle, Furbetto (del quartierino), in "Lingua Italiana d'Oggi" III, 2006, pp. I49-I53.

- Giovanardi-Gualdo-Coco 2008: Claudio Giovanardi, Riccardo Gualdo, Alessandra Coco, IngleseItaliano 1 a 1. Tradurre o non tradurre le parole inglesi? Nuova edizione riveduta e ampliata, San Cesario di Lecce, Manni, 2008.

- Gualdo 20Io: Riccardo Gualdo, Quanto pesa l’inglese? Anglicismi nella vita quotidiana e proposte per la coabitazione, in Per litaliano. Saggi di storia della lingua nel nuovo millennio, Roma, Aracne, 20 o.o.

\section{Cita come:}

Riccardo Gualdo, $A$ /badge ci siamo ormai abituati, a beggiare e alla beggiatura no, "Italiano digitale", 2020, XIV, 2020/3 (luglio-settembre)

DOI: $10.35948 / 2532-9006 / 2020.4375$

\section{Copyright 2020 Accademia della Crusca}

Pubblicato con licenza creative commons CC BY-NC-ND 